\title{
Knowledge, Attitude and Practice of Medical Incident Reporting Among Healthcare Professionals: A Study of Midigo Health Centre IV
}

\author{
Article by Omona Kizito \\ Ph.D in Healthcare Management, Texila American University, Uganda \\ E-mail: kizitoomona@gmail.com
}

\begin{abstract}
Introduction: Medical incident reporting is a key tool for improving patient safety in healthcare, hence improved quality of care. The better the knowledge, attitude and practice of this concept, the better the quality of care. The perceived knowledge, attitude and practice are still low in many Uganda healthcare facilities. Many of the healthcare providers have, therefore, limited their scope to maternal death audit and reporting or perinatal death reporting and to a greater extent Adverse Event Following Immunization (AEFI). This problem of perceived low knowledge, attitude and practice of incident reporting is coupled with the poor institutional culture to persistently and inadequate support healthcare professionals to report incidents. Medical incident reporting is, therefore, the single most powerful tool for developing and maintaining an awareness of risks in healthcare practice, hence a cornerstone to improved patient safety and improved quality of service delivery.

Objectives: The study objectives were as follow; to assess the level of knowledge about medical incident reporting among healthcare professionals, by April, 2016 and determine their attitude towards medical incident reporting. The researcher also set out to ascertain the practice and the extent to which medical incident reporting is practiced in Midigo Health Centre IV, as well as, establishing the factors affecting medical incident reporting in the said healthcare facility.

Methods: The study was a cross sectional study of knowledge, attitude and practice of medical incident reporting among Healthcare Professionals in Midigo health centre IV. It was both qualitative and quantitative; with a sample of 44healthcare professionals interviewed using structured questionnaires. The questionnaire was pre-tested. Analysis of result was done using computer packages called Statistical Package for Social Sciences (SPSS) and Microsoft excel. Ethical considerations in research were observed.

Results: The cadre of the respondents were; Medical officers $-4.5 \%$, Clinical officers $6.8 \%$, Nurses $-43.2 \%$, Midwives $-11.4 \%$, Theatre staffs $-9.1 \%$, Laboratory staffs $-6.8 \%$ and other staffs $-18.2 \%$. The response rate was $100 \%$. The level of knowledge about medical incident reporting among healthcare professionals in Midigo HC IV was at 84.1\%, by April 2016. Much as there was no statistical significance between cadre of staffs and extent of knowledge, $p$-value $>0.39$, the only cadres that had excellent knowledge on medical incident reporting were nurses (75\%) and clinical officers (25\%). The rest of the staffs either had average knowledge or fair knowledge or no knowledge at all. The healthcare professionals had strong positive attitude towards medical incident reporting and this was at $97.7 \%$. By April, 2016, the practice of medical incident reporting was at $72.6 \%$ with the majority of these respondents (up to 50\%), having participated in reporting three times or more for the last 5years. The major factors that facilitated the respondents to report were; Strong positive feeling to participate and improve patient safety and respondents were knowledgeable (educated) about medical incident reporting. Other minor factors like ability of respondents to get feedback on reported incidents and strong institutional culture of reporting did not make strong contribution towards the practice of reporting. However, the major barriers were; respondents didn't know where and how to report, coupled with weak institutional culture of reporting incidents. Surprisingly, fear of consequence of reporting did not in any
\end{abstract}


Texila International Journal of Medicine

Volume 4, Issue 2, Dec 2016

way hinder any respondent from reporting. In other words, it was not a reason for them not to participate in medical incident reporting.

Conclusion: Medical incident reporting still remains a key tool in improving patient safety. The greater the practice of reporting, the better; as evident by the strong positive feeling towards medical incident reporting, in this research finding. Educating professionals on incident reporting and strong positive individual feeling to improve safety have remained the major factors facilitating medical incident reporting. Likewise, lack of knowledge on where and how to report, coupled with weak institutional culture of reporting have remained the major barriers to practicing medical incident reporting.

\section{Introduction}

This section covers introduction to the study topic, background to the study area, statement of the problem, research question, conceptual framework, research objectives and justification for the study in that order

\section{Introduction to the study topic}

Medical incident reporting in healthcare is presumed to have helped a lot in the reduction of healthcare associated errors/risks. According to British journal of anesthesia, Critical incident reporting, key in improving safety, is under-utilized in healthcare systems (Mahajan, 2010). Anderson Janet E., Naonori Kodate, Rhiannon Walters And Anneliese Dodds (2013) argued that incident reporting is well accepted in safety critical industries such as aviation, as a method for improving safety, and that it is now well established in healthcare in many countries, including the United Kingdom. Medical incident reporting seems to have proven effective in improving patient safety in healthcare. It is presumed that the already over-burden healthcare professionals, in healthcare setting, may not pay keen attention in each and everything in the healthcare setting. One such area that appears to be under looked by many healthcare professionals is medical incident reporting, itself. This argument has been supported by the work of earlier researchers on patient safety and medical errors among Italian hospital Physicians (Domenico, F., Paolo, R., Aida, B., Claudia, P. And Maria, P., 2012).

Studies have shown that Poor staffing levels hinder effective communication amongst health workers, leading to provider fatigue and creating an environment for medical incidents. Similarly, poor organizational safety culture, with resultant under-reporting of medical incidents and predominantly punitive responses to errors by health workers seem to have lowered health workers" attitudes to report (Nabudere, H., Asiimwe, D. and Semakula, D., 2014). The practice of medical incident reporting goes down as the attitude lowers and the end result is that quality of care is compromised.

In their study on the perceived effectiveness of incident reporting in improving safety, among healthcare professionals, Anderson et al (2013) found that indeed incident reporting was perceived to have a positive effect on safety. They further stressed that this positive effect did not only lead to changes in care processes but also change the staff attitudes and knowledge. In fact, they concluded that incident reporting can be a powerful tool for developing and maintaining an awareness of risks in healthcare practice, hence patient safety improvement.

Other studies have shown that healthcare professionals appear reluctant to report adverse incidents to a senior member of staff. Doctors were more unwilling than nurses or midwives to report adverse incidents to a senior staff member. Incident reporting was most likely when the incident involved the deviation from a protocol and when the outcome for the patient was bad (Lawton, R. and Parker, D., 2002). The argument is that reporting must be a routine selfdriven practice among healthcare professionals. Omona (2015, pp.34-35), in his study to determine the level of health workers" engagement in patient safety in Kitgum general hospital, found that the majority of the health workers (77.3\%) never reported the medical incidents they encountered. The major reason (50.8\%) for not reporting was because they did 
not know where to report, owing to poor reporting culture in the health facility. In Uganda, however, the commonly reported medical incidents are limited to maternal death, perinatal death and Adverse Reaction Following Immunization (ARFI) or in emergency out breaks.

\section{Background to the study area}

Midigo health centre IV is a government health facility, located in Yumbe district (West Nile), specifically located in Midigo Sub County. It is the only health centre IV in the district and has a total bed capacity of 73beds ( $\mathrm{MoH}, 2015)$. Midigo Sub County has a total population of 37,100people which is $7.64 \%$ of the total population of Yumbe district of 485,582 persons (UBOS, 2015).

The health centre has been partly supported for a long period of time (about 15years) by a Church based development partner, Calvary Chapel of Midigo. Calvary Chapel contributes about $35 \%$ of the healthcare professional staffs to the health facility, alongside with other supports including monthly procurement of medicines and other medical supplies.

\section{Statement of the problem}

Medical incident reporting appears to be a very important practice in improving patient safety in healthcare. In Uganda, many healthcare professionals appear to be relaxed except in areas of maternal death and perinatal death reporting, adverse event following immunization and a few others, where some reporting is practiced.

The main problems are presumed to be the knowledge gaps, negative attitude towards reporting and low level of practice of actual reporting of medical incidents among healthcare professionals. These problems are presumed to cut across the various health facilities countrywide. Midigo health centre IV is just one of the few health facilities, among others. The cause of these problems are probably; lack of adequate professional medical development (PMD)/Continuous medical education (CME) on the subject matter, fear of punitive consequences of reporting, lack of motivation to do so (poor reporting culture) and perceived lack of time due to understaffing, among others. The solutions to these problems are lying in addressing the mentioned causes.

The effects of these problems are increased healthcare risk for patients and compromised quality of healthcare services. These problems are thought of by the researcher to deserve special attention and have, therefore, prompted the researcher to find out the magnitude of such in the Midigo health centre IV.

\section{Research questions}

This study sought to answer the following research questions;

I. What is the level of knowledge about medical incident reporting among healthcare professionals in Midigo Health Centre IV?

II. What is the attitude of healthcare professionals in Midigo Health Centre IV towards medical incident reporting?

III. To what extend is medical incident reporting practiced in Midigo Health Centre IV?

IV. What are the factors affecting medical incident reporting in Midigo Health Centre IV?

\section{Conceptual framework}

The dependent variable or variable of primary interest, as shown in the model, is medical incident reporting while the independent variables are healthcare professional knowledge, attitude and practice of medical incident reporting. The co-founders affect the out outcome of incident reporting. 


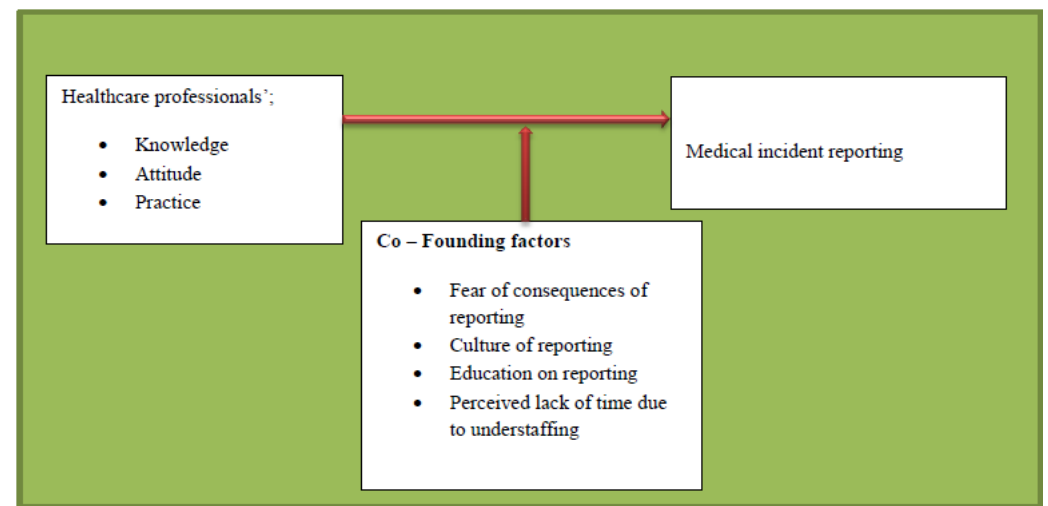

Figure 1: Conceptual model

\section{Objectives of the study}

The study had both broad objective and specific objectives, under sub-sections below;

\section{Broad objectives}

The broad objective is to contribute to improved patient safety in healthcare through creating awareness on the need for medical incident reporting among healthcare professionals in Midigo Health Centre IV

\section{Specific objectives}

There are four specific objectives for this study. These are as below stated;

I. To assess the level of knowledge about medical incident reporting among healthcare professionals in Midigo Health Centre IV, by April 2016

II. To determine the attitude of healthcare professionals in Midigo Health Centre IV towards medical incident reporting, by April 2016.

III. To ascertain the practice and the extent to which medical incident reporting is practiced in Midigo Health Centre IV, by April 2016.

IV. To establish the factors affecting medical incident reporting in Midigo Health Centre IV, by April 2016.

\section{Justification of the study}

However the study findings, if utilized, would help achieve the following;

I. The healthcare stakeholders will appreciate the relationship between patient safety and medical incident reporting. If found to be a positive relationship (that improves patient safety in healthcare), the finding will be used to make decisions to improve the weak areas of the healthcare settings. Some of the weak points could be; the Institution may have a less supportive environment for event reporting that does not protect the privacy of staffs reporting, reports received from a narrow range of personnel, summaries of reported events may not be disseminated in a timely fashion and less structured mechanism for reviewing reports and developing action plans (Agency for Healthcare Research and Quality, 2014)

II. The study will add to the existing body of knowledge as a future reference material and for any researcher who may wish to do similar study elsewhere.

\section{Literature review}

\section{Introduction}

This chapter has been laid down in the following order; Introduction, level of knowledge about medical incident reporting among healthcare professionals, attitude of healthcare professionals towards medical incident reporting, practice and the extent to which medical incident reporting is practiced in healthcare settings and factors affecting medical incident reporting in that order. Conclusion of this chapter was made last in the sub-section. Findings 
of many scholars related to this subject matter were sought and reviewed as per citations in this chapter.

\section{Knowledge about medical incident reporting among healthcare professionals}

World Health Organization (WHO) defined Incident reporting as a process used to document occurrences that are not consistent with routine hospital operation or patient care. Similarly, patient safety was defined as freedom from accidental injuries during the course of medical care; activities to avoid, prevent, or correct adverse outcomes which may result from the delivery of health care (WHO, 2009). Incident reporting is viewed at as a measure to improve patient safety in health care, by many scholars. In fact, WHO argued that for several reasons, the effectiveness of incident reporting is limited. These reasons, among others, include fear of punitive action, reluctance of non-physicians to report incidents involving physicians, lack of understanding of what a reportable incident is and lack of time to report medical incidents (WHO, 2009)

Marilyn, J. K., Sue, M. E., Brian, J. S. and Jesia, G. B. (2004), in their study on attitudes of doctors and nurses towards incident reporting found that knowledge gap was one of the factors that negatively affected incident reporting. They argued that this gap can be adequately addressed through healthcare professional education. Their view was supported in another study. After the study on the effect of incident reporting following patient safety education, it was concluded that patient safety education can have immediate and long-term positive effects on knowledge, skills and attitudes, and modestly influence the reporting behavior of resident healthcare professionals (Jansma, J. D., Cordula, W., Reinier, W. t. K. and Arnold, B. B., 2011).

In a study on healthcare professionals " views of the effectiveness of incident reporting, Anderson et al, (2013), found that incident reporting had a very strong positive effect on patient safety, not only by leading to changes in care processes but also by changing staff attitudes and knowledge. In another study on engagement in patient safety, Omona (2015) found that health workers in Kitgum general hospital were $4.5 \%$ more aware about patient safety than the patients. This awareness did not have strong engagement to improve safety in terms of what health workers do, such as incident reporting.

In a study on factors influencing incident reporting in surgical care, it was found that nurses were more knowledgeable than doctors when it comes to incident reporting (Kreckler, S., Catchpole, K., McCulloch, P. and Handa, A., 2009)

Some knowledge gaps still exist, definitely, in incident reporting systems in many countries. Anja, H., Brunsveld-Reinders, M., Sesmu, A., Rien, D. V. and Evert, D. J., (2015) in their study pointed out that many of the incident reporting systems could not meet WHO criteria. No attention was given to analyzing incidents reported or giving feedback on the reported incidents. This resulted in just a simple administrative report system, rather than the much desired instrument for change of practice and increase of quality of care. Incident reporting system can only effectively contribute to improve patient safety and quality of care if more attention is given to analyzing incidents and feedback.

Again, according to Patient Safety Network (PSNet), a successful incident reporting system should be easy to use and must contain the four components. These are;

- Institution must have a supportive environment for incident reporting that protects the privacy of staff who report occurrences

- Reports should be received from a broad range of personnel

- Summaries of reported events must be disseminated in a timely fashion

- Lastly, a structured mechanism must be in place for reviewing reports and developing action plans

Much as incident reporting utilization can be a cornerstone of a positive safety culture within the institution, such institutions need to resist the temptation to encourage incident reporting without a concrete plan for following up on the reported events (PSNet, 2014) 
Texila International Journal of Medicine

Volume 4, Issue 2, Dec 2016

Therefore, all in all, much as there may be few knowledge gaps in medical incident reporting, the greatest problem is different, as pointed out by other scholars. In a study on Culture of blame in the National Health Service in United Kingdom (UK), it was pointed that 'The single greatest impediment to error prevention is that we punish people for making mistakes' This is what makes incident reporting weak (Radhakrishna, 2016). This view was supported by Vincent (2011) who argued that the culture of blaming and disciplining in case of medical incidents are ineffective responses to most safety problems.

\section{Attitude of healthcare professionals towards medical incident reporting}

In a study on the views of junior doctors on reporting incidents, in United Kingdom (UK), it was noted that these category of healthcare professionals (junior doctors) participated less in incident reporting. This was not because they had negative attitude towards the practice, but it was predominantly due to lack of supporting environment to report (Hooper, P., Kocman, D., Carrl, S. and Tarrant, C., 2015).

This argument was supported by another study on attitudes of doctors and nurses towards incident reporting. It was found that Nurses reported more habitually than doctors as a result of culture which provided directives in the nursing arena as opposed to such environment for doctors (Marilyn et al., 2004).

In fact, according to Jansma et al. (2011), patient safety education can have immediate and long-term positive effects on knowledge, skills and attitudes, and modestly influence the reporting behavior of healthcare professionals.

In a similar study on barriers and motivating factors in reporting incidents of assault, it stated that personal attitudes may affect reporting, especially if individual reporting thinks that reporting is optional or he or she sees reporting as "taking on a battle." Other attitudes such as seeing personal effort to report as not worth, because of the minimal expectation of a positive outcome, was also noted. Again, the fear of being blamed stood out strongly as a perception that deter healthcare professionals from reporting medical incidents (Gifford and Anderson, 2010) Furthermore, they argued that individual attitudes such as; belief that reporting is optional, Self-blame and fear of embarrassment quite often negatively affect incident reporting.

The ultimate goal of incident reporting is to improve on patient safety, by way of reducing medical errors. It has, therefore, been argued that healthcare professionals who get involved in adverse incidents or those who seem to have caused adverse incident, often feel guilty, shameful and develop burnout. This is common among junior healthcare professionals and in a way could negatively affect their attitude towards medical incident reporting (Naonori and Anneliese, 2008). Vincent (2011) argued that the culture of blaming and disciplining healthcare professional on such account is an ineffective response to most safety problems. In another study by Polisena, J., Gagliardi, A., Urbach, D., Tammy, C. and Michelle, F., (2015), findings indicated that fear of punishment, uncertainty of what should be reported and how incident reports will be used and time constraints to incident reporting are common barriers to incident recognition and reporting. For surely, healthcare professionals who have fear of punishment associated with incident reporting, will be negatively affected when it comes to medical incident reporting.

In a study about attitudes and barriers to incident reporting, it was found that both doctors and nurses believe they should report most incidents, but nurses were found to do so more frequently than doctors. In their conclusion, to improve incident reporting, especially among doctors, clarification was needed of which incidents should be reported, the process needed to be simplified and feedback given to the reporters (Evans et al., 2006). In a study on patient safety and medical errors among Italian hospital physicians, Domenico et al., (2012) concluded that greater efforts were needed. These efforts were to facilitate the translation of positive attitudes towards patient safety into appropriate practices that have proven to be effective in the reduction of medical errors (increased patient safety). 


\section{Practice and the extent to which medical incident reporting is carried out in healthcare settings}

The practice of incident reporting needs to be as inclusive as possible, with most of the stakeholders getting involved. In a study on anesthesia related incidents, it was made that post-operatively, quality assurance officer would constitute a team to do the review of what went wrong. In that review meeting, staff members would be encouraged to provide comments and suggestions for improvement. This meeting was regularly done for every morbidity-mortality related cases and the incidents were discussed in detail with the staff members involved in the process describing the sequence of event and suggesting a number of corrective strategies to avoid similar incidents re-occurring (Haller, G., Courvoisier, D. S., Anderson, H. and Myles, P. S., 2011).

The extent of incident reporting depend the level of knowledge and positive attitudes towards patient safety concept, among healthcare professionals. Therefore, the more someone is educated on patient safety, the more the ability and willingness to report incidents. In fact, positive changes in knowledge, skills and attitudes would be paramount after such education course (Jansma et al., 2011)

\section{Factors affecting medical incident reporting}

Medical incident reporting practice is still lower than expected in many Ugandan healthcare facilities. A number of factors are negatively affecting this practice, which would otherwise improve quality of care.

In a similar study, it was found that fear of punishment, uncertainty of what should be reported and how incident reports will be used and time constraints to incident reporting were among the common barriers to incident recognition and reporting. It was concluded that strategies to improve error reporting were; the use of an electronic error reporting system, increased training of healthcare professionals and giving feedback to frontline healthcare professionals about the reported error (Polisena et al., 2015). However, Vincent (2011) argued that the culture of blaming and disciplining in case of medical incidents are ineffective responses to most safety problems. They are ineffective in the sense that they markedly affect improvement strategies but encourage defensive tactics by healthcare professionals.

These findings were not far different from those found by Gifford and Anderson (2010). For them, the major factors were; feeling that reporting was optional, fear of blame and embarrassment, among others. Similarly, Marilyn et al. (2004) found the common barriers to reporting incidents included; time constraints, unsatisfactory processes, deficiencies in knowledge, cultural norms, inadequate feedback, beliefs about risk and a perceived lack of value in the process.

\section{Conclusion}

According to (Julius, C. P., Thierry, G. and Peter, J. P., 2013), Incident Reporting System (IRS) is and will continue to be an important influence on improving patient safety. In their review on IRS, they pointed at four major advantages (uses) of incident reporting as follows;

- To identify local system hazards

- To aggregate experiences for uncommon conditions

- Used to share lessons within and across organizations (health institutions)

- Used to increase patient safety culture

When this concept is not well understood by healthcare professionals, the practice might markedly be affected negatively. Similarly, when it is well understood, the practice would be perfect hence improved patient safety and quality of healthcare. 
Texila International Journal of Medicine

Volume 4, Issue 2, Dec 2016

\section{Research methodology}

\section{Introduction}

This chapter illustrates the study area, study design, study population, study unit, sample size estimation, sampling techniques, the variables for the objectives and their indicators, data collection and study instruments, data analysis and presentation methods, quality controls, ethical considerations, limitations of the study and plans for dissemination of results.

\section{The study area}

The research was done in Midigo Health Centre IV, located in Midigo sub county, in Yumbe district (West Nile region), Uganda.

\section{Study duration}

The study took a period of three months (February - April), in the year 2016

\section{The study type/design}

This was a descriptive, cross sectional study of Knowledge, attitude and practice of medical incident reporting among healthcare professionals in Midigo Health Centre IV. The study took both qualitative and quantitative dimensions.

\section{Study Population}

The study population was all the healthcare professionals (both medical workers and the support staffs) in Midigo health centre IV.

\section{Study Units}

These were healthcare professionals present in the health facility at the time of the study/during the period of the study.

Inclusion Criteria: All the healthcare professionals present in the health facility at the time of the study were free to participate in the study. This included all those healthcare professionals who directly or indirectly got involved in medical incident reporting.

Exclusion Criteria: All healthcare professionals who were not physically present in the health facility (Such as those on study leave or sick leave) at the time of the study or those who were unwilling to participate in the study were excluded. Again, students who were for internship/industrial training were excluded.

\section{Sample size estimation}

Since the population size of healthcare professionals in Midigo health centre IV was known (49health workers), the formula for calculation of sample size was used to compute the sample size.

Therefore, using the formula;

$$
n=\mathrm{N} /\left[\mathbf{1}+\mathbf{N}(\mathrm{e})^{2}\right]
$$

Where $n$ is the sample size, $\mathrm{N}$ is the population size (Total population of the study), e is the level of precision (level of confidence) and 1 is a constant

Hence; $n=49 /[1+49(0.052)]$

$$
\begin{aligned}
& n=49 / 1.1225 \\
& =43.6526 \\
& \sim 44 \text { health workers }
\end{aligned}
$$

\section{Sampling techniques}

The techniques used were both probability and non-probability sampling. For healthcare professional cadres that were very few in the health facility and yet their knowledge, attitude and practice is key in medical incident reporting, purposive sampling technique was used. These categories of healthcare professionals were medical officers, clinical officers, midwives, theatre staffs and laboratory staffs. The likelihood of missing their views in this 
research was very high because of their low numbers and therefore the researcher purposely selected them to be included in the sample.

Simple random sampling technique was then used to select the remaining categories of staffs such as nurses and nursing assistants, among others. This was done until the entire 44respondents healthcare professionals were obtained.

\section{Variables and Indicators for the objectives}

The variables and indicators for all the four specific objectives have been described in the sub-sections below. The healthcare professionals" knowledge, attitudes and practices were taken as the independent variables while medical incident reporting was the dependent variable. Medical incident reporting was taken to be dependent on whether or not the healthcare workers were knowledgeable about it, have positive attitudes towards it or practice it in their day to day service delivery.

Variables and indicators for objective I: To assess the level of knowledge about medical incident reporting among healthcare professionals in Midigo Health Centre IV.

The variable used for this objective was healthcare professional knowledge of medical incident reporting. The indicator was the „YES" or „No " responses given by the respondent, on whether or not they knew about the subject matter. This was used as a proxy measure of the respondents" knowledge on the subject matter.

Variables and Indicators for objective II: To determine the attitude of healthcare professionals in Midigo Health Centre IV towards medical incident reporting

The independent variables for this objective are healthcare professionals " attitudes (categorized as negative or positive). The indicators were as follows; the respondents who accepted that medical incident reporting should be removed from healthcare practice were taken as those with negative attitude towards the practice. Similarly, those who want the practice to continue, even at higher level, were taken as respondents with positive attitudes towards the practice.

Variables and Indicators for objective III: To ascertain the practice and the extent to which medical incident reporting is practiced in Midigo Health Centre IV

The independent variables for this objective were „the practice of incident reporting " and the „extend of this practice. " This was measured against the number of respondents who had ever practiced any incident reporting within a period of one year. Similarly, the extent of the practice was measured against the frequency of such practice.

Variables and Indicators for objective IV: To establish the factors affecting medical incident reporting in Midigo Health Centre IV

For the respondents who practiced medical incident reporting in Midigo HC IV, the variables were the stated factors which made them to do so. Similarly, for those who did practice reporting, the stated factors that prevented them from doing so were considered as variables.

\section{Data collection tools and techniques}

The data collection tool used in this study was purely questionnaires. Whereas, the data collection techniques were used in was self-administering of semi-structured questionnaires to the respondents. Reasonable amount of time to fill the questionnaire was agreed upon between the research assistant and the respondent.

\section{Data entry, analysis and presentation methods}

Data entry and analysis was made using two computer softwares; Statistical Package for Social Sciences (SPSS) and Microsoft excel. The researcher did the data entry and analysis by himself, in consultation with an experienced data analyst.

Pie charts, tables, bar graphs and descriptive methods for the qualitative components were used as data presentation methods for this research. Simple frequency tables and cross tabulations were drawn to present results. 
Texila International Journal of Medicine

Volume 4, Issue 2, Dec 2016

\section{Quality controls}

For quality assurance purpose, the following quality control measures were employed;

I. Pre-testing of the questionnaires was done before actual data collection was made. Changes to the questionnaires were made as appropriate, after the pre-testing exercise.

II. Other ways used to improve quality of data included; training of the research assistant, close field supervision by the researcher and a careful data validation at the point entry and analysis. The researcher took particular interest in doing this validation and personally did the analysis.

\section{Ethical considerations}

The following ethical considerations were taken care of;

I. Permission was sought from office of the District Health Officer (DHO), of Yumbe district local government, as well as the In-Charge of Midigo HC IV.

II. Informed consents from the respondents were sought before engaging them. Confidentiality of data collected was maintained.

III. Participation of the respondents was purely voluntary. Respondents were free to pull out of the study, for whatever reason(s) and their decisions to do so were highly respected.

\section{Limitations to the study}

The study had the following limitations;

I. The researcher, at the time of the study, was a high profile healthcare professional in the same health facility (Midigo HC IV). Therefore, his direct involvement could induce respondents" bias in filling the questionnaires. To solve this concern, an independent research assistant was used to collect data.

II. Not all the healthcare professionals who participated in the study were directly/equally exposed to medical incident reporting. Therefore, their level of knowledge in the subject matter varied markedly from the others who were exposed. To address this concern, key terms used were defined in the introduction to the questionnaire, to help them understand the subject matter before filling their questionnaires. Examples were also given about certain key terms.

\section{Plan for dissemination of results}

The result of this study was disseminated to all the staffs of Midigo HC IV. Part of it was shared with the office of District Health Officer (DHO), the District Health Team (DHT) and office of the Chief Administrative Officer (CAO) of Yumbe district local government. This work is also planned for dissemination across Uganda and the world at large, by way of publishing it.

\section{Results, analysis and presentation}

\section{Introduction}

The results of the analysis of this study have been discussed as under. Tables, pie charts and bar graphs were used. Percentages and frequencies were also used to present results. The respondents were healthcare professionals working in Midigo health centre IV, in Yumbe district, in the year 2016.

Socio-demographic characteristics of the respondents: There were 44respondent healthcare professionals, with response rate of $100 \%$. The male respondents were 26 in number, constituting $59.1 \%$ of the total respondent whereas the female respondents were 18 in number (40.9\%). The majority of the respondents, 52.3\%, were of older age group (More than 35years old). $45.5 \%$ of the respondents were in the middle age group (25-35years old) while $2.3 \%$ were in the younger age group, less than 25years old.

The cadre of the respondents were as follows; Medical officers $-4.5 \%$, Clinical officers $6.8 \%$, Nurses $-43.2 \%$, Midwives $-11.4 \%$, Theatre staffs $-9.1 \%$, Laboratory staffs $-6.8 \%$ 
and other staffs $-18.2 \%$. Therefore, nurses were the majority followed by the other category of staffs (Cleaners, Security officers, Public health specialist, recorder staffs, store assistants and others).

\section{Level of knowledge about medical incident reporting among respondents healthcare professionals}

The knowledge of the respondents was first assessed on medical incident reporting. Later, the extent of this knowledge was assessed for each respondent who knew about it.

To assess the level of knowledge of the about medical incident reporting in the healthcare facility, the respondents were asked whether or not they knew medical incident reporting before the study. „Yes" or "No" responses were given. The result has been shown in the table below.

Table1: Whether respondent knew about medical incident reporting or not

\begin{tabular}{|c|l|l|l|l|l|}
\hline \multicolumn{6}{|c|}{ Respondent knew about Medical Incident Reporting } \\
\hline Valid & Yes & Frequency & Percent & Valid Percent & Cumulative Percent \\
\hline & No & 7 & $84.1 \%$ & $84.1 \%$ & 84.1 \\
\hline & Total & 44 & $15.9 \%$ & $15.9 \%$ & 100.0 \\
\hline
\end{tabular}

From table 1 above, the majority of the respondents, 84.1\% knew about medical incident reporting before the study. Only 7respondents, constituting $15.9 \%$ did not know about the subject matter.

Based on this, the researcher concluded the level of knowledge about medical incident reporting among healthcare professionals in Midigo HC IV to be 84.1\%, by April 2016.

Extent of the knowledge among respondents who knew about medical incident reporting: The respondents who knew about medical incident reporting were then categorized into three; category one were those who had excellent knowledge, category two were those who had average knowledge while category three were those who had fair knowledge. The results of the findings were as presented in figure below.

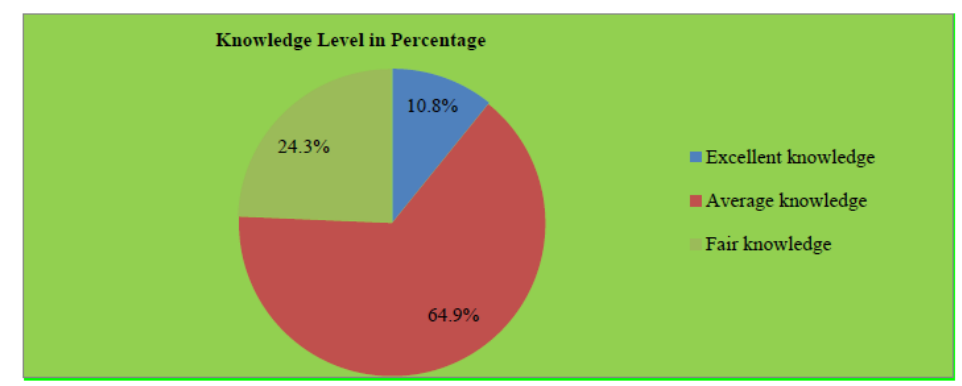

Figure 2: Level of knowledge among respondents who knew about medical incident reporting

Figure 2 above shows that out of the $84.1 \%$ respondents who knew about medical incident reporting; only $10.8 \%$ had excellent knowledge. The majority $(64.9 \%)$ had average knowledge.

A cross tabulation between extent of knowledge and cadre of staff was done. The summary results are shown in table 2 below. 


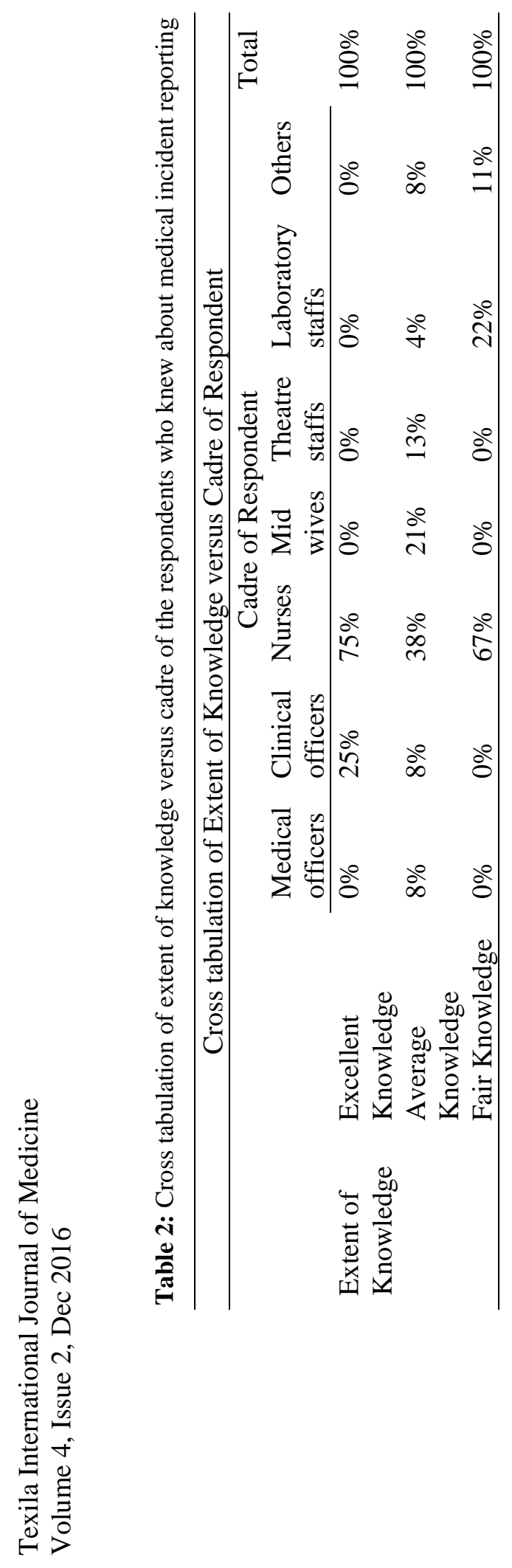


From table 2 above, nurses were the single most important cadre of healthcare professionals with excellent knowledge (75\%) followed by clinical officers (25\%). However, this finding was not statistically significant, $X 2(12, N=44)=12.7, p=.39$, it was by chance that these cadres had higher knowledge level. Other factors could have played, including exposure to incident reporting following their previous work environments. Probably, a security officer may not have adequate knowledge about medical incident reporting to the level of a nurse or a midwife, much as they are all health workers. This is because the environment where a security officer works may not give him/her enough exposure.

Most of the staffs in Midigo health centre IV had average knowledge and this cut across all cadres of healthcare professionals in the facility.

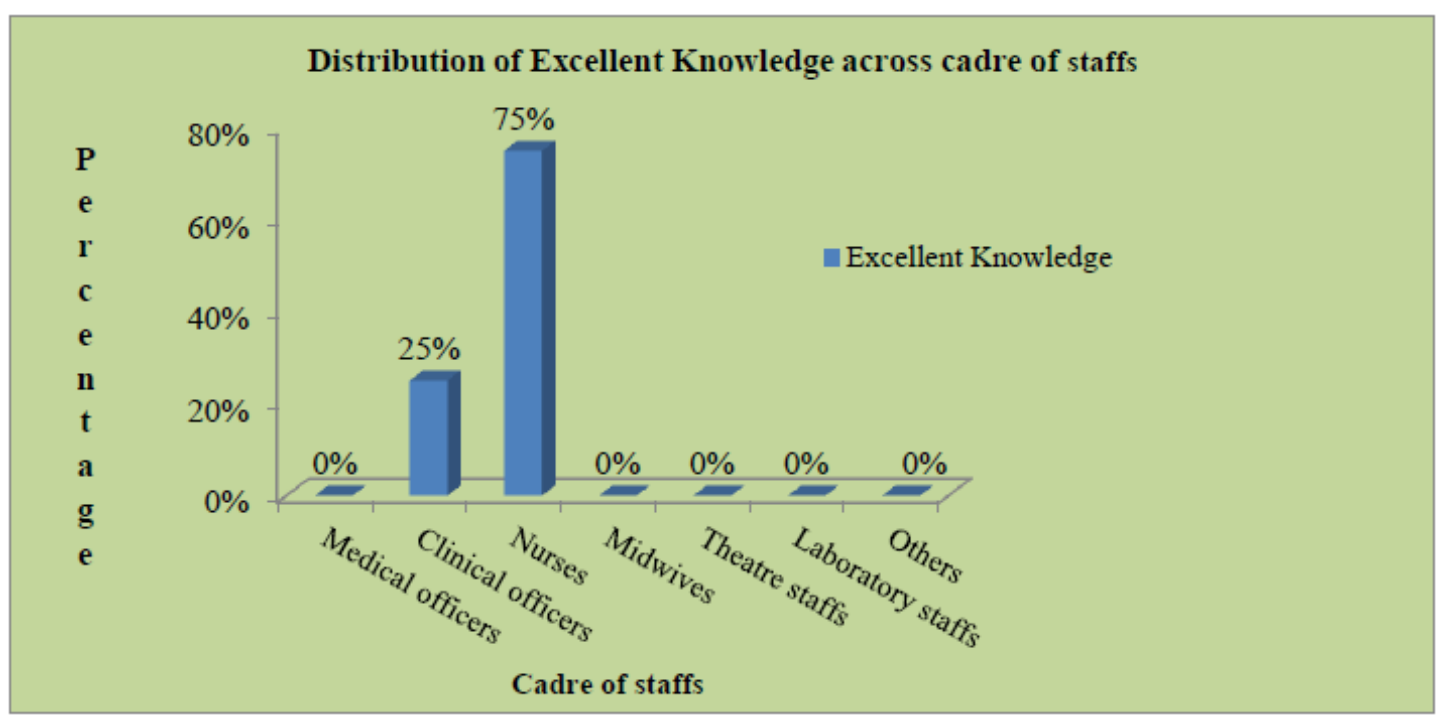

Figure 3: Distribution of excellent knowledge among respondents who knew medical incident reporting

Figure 3 above, shows that a healthcare professional in Midigo health centre IV who excellently knew about medical incident reporting was either a nurse, with $75 \%$ chance or a clinical officer, with $25 \%$ chance respectively.

\section{Attitude of healthcare professionals in Midigo Health Centre IV towards medical incident reporting}

The respondents were asked a strong negative statement and a strong positive statement about the need to used medical incident reporting to improve patient safety. Asking the respondent to state whether incident reporting was a „waste of time, and must be discouraged immediately" showed a strong negative feeling about the exercise. This was taken by the researcher as a negative attitude towards medical incident reporting. Similarly, asking the respondent to state whether incident reporting ,improves patient safety, and therefore must be encouraged" showed a strong positive feeling of the respondent about the exercise. This was taken as a positive attitude towards medical incident reporting.

Table 3: Attitude of healthcare professionals toward medical incident reporting

\begin{tabular}{llllll}
\hline \multicolumn{6}{l}{ Attitude of Respondent About Medical Incident Reporting } \\
\hline & Frequency & Percent & Valid Percent & $\begin{array}{l}\text { Cumulative } \\
\text { Percent }\end{array}$ \\
Valid & & & & 2.3 \\
& Strong Negative Attitude & 1 & 2.3 & 2.3 & 2.3 \\
& Strong Positive Attitude & 43 & 97.7 & 97.7 & 100.0 \\
& Total & 44 & 100.0 & 100.0 & \\
\hline
\end{tabular}


Texila International Journal of Medicine

Volume 4, Issue 2, Dec 2016

From table 3 above, the majority of the respondents had strong positive attitude toward medical incident reporting, standing at $97.7 \%$. The negative feeling about the practice, herein referred to as attitude, was at $2.3 \%$

This result has been graphically presented as below;

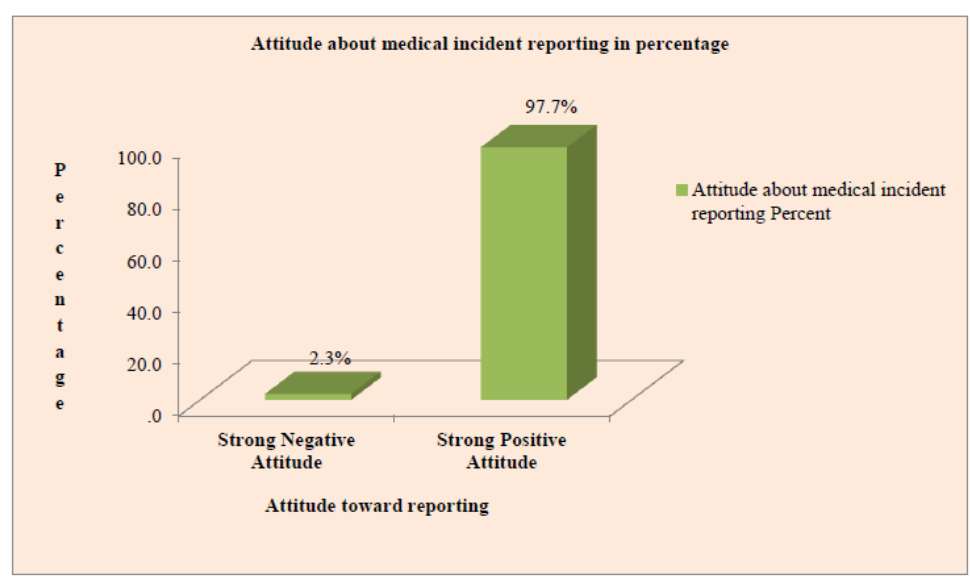

Figure 4: Attitude of healthcare professional in Midigo health centre IV towards medical incident reporting

Figure 4 above showed that positive attitude towards medical incident reporting in Midigo health centre IV was at $97.7 \%$ while negative attitude was at $2.3 \%$.

\section{The practice of medical incident reporting in Midigo Health Centre IV}

The respondents were asked where or not they had ever participated in reporting incident over the last 5years. „Yes" or "No" responses were solicited from them and the researcher based on these responses, as a proxy measure, to ascertain the level of practice of medical incident reporting in the facility. The findings were summarized in graphic form as below.

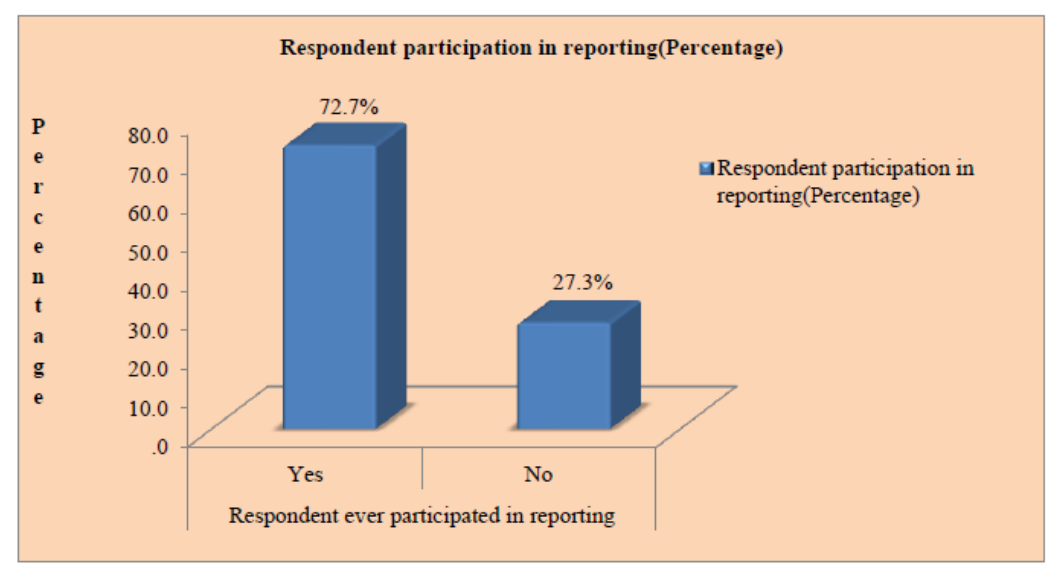

Figure 5: Practice of medical incident reporting over 5years period

From figure 5 above, the majority (72.7\%) of the respondent healthcare professionals in Midigo health centre IV ever participated in the medical incident reporting over the last 5years while $27.3 \%$ never did so. The level of practice was concluded based on the „Yes" response, which was $72.7 \%$.

The extent of practice of medical incident reporting among respondents who ever participated in medical incident reporting over the last $5 y e a r s:$ The respondents who had ever participated in incident reporting over 5years period were asked how many times they participated within the said time frame. The response categories solicited were; Once, Twice and Three times or more. The results were summarized in figure 6 below. 


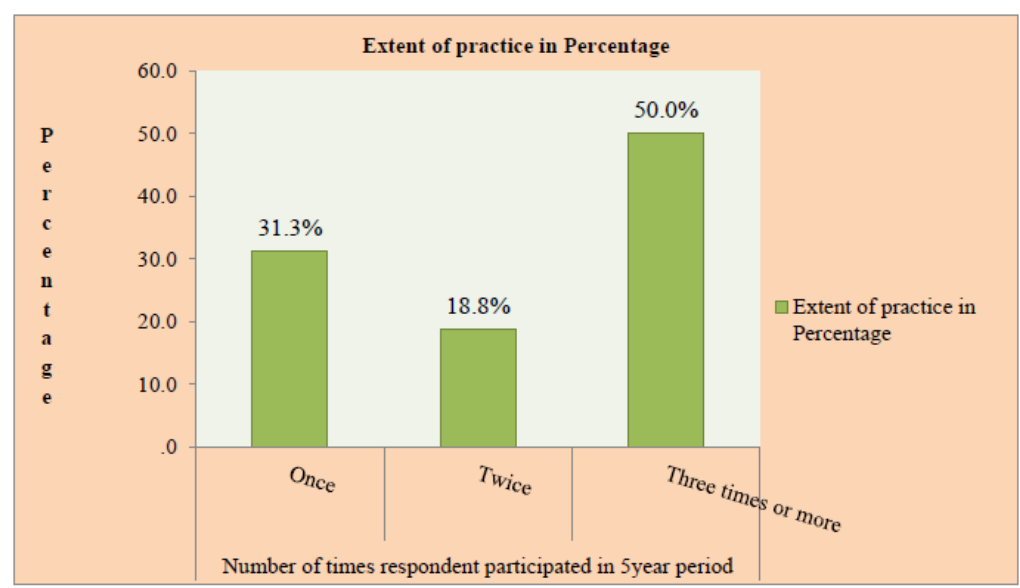

Figure 6: Extent of practice of medical incident reporting in a 5year period

Figure 6 above shows that out of the 72.6\%respondent healthcare professionals who ever participated in incident reporting in a 5year period, the majority $(50.0 \%)$ had participated three times or more. This was followed by those who participated once, constituting 31.3\%.

Relationship between extent of practice of medical incident reporting over a 5year period and cadre of respondent: The researcher proceeded to examine the relationship between extent of practice in a 5year period and cadre of respondent. A cross tabulation was done and the findings were shown figure 7 below

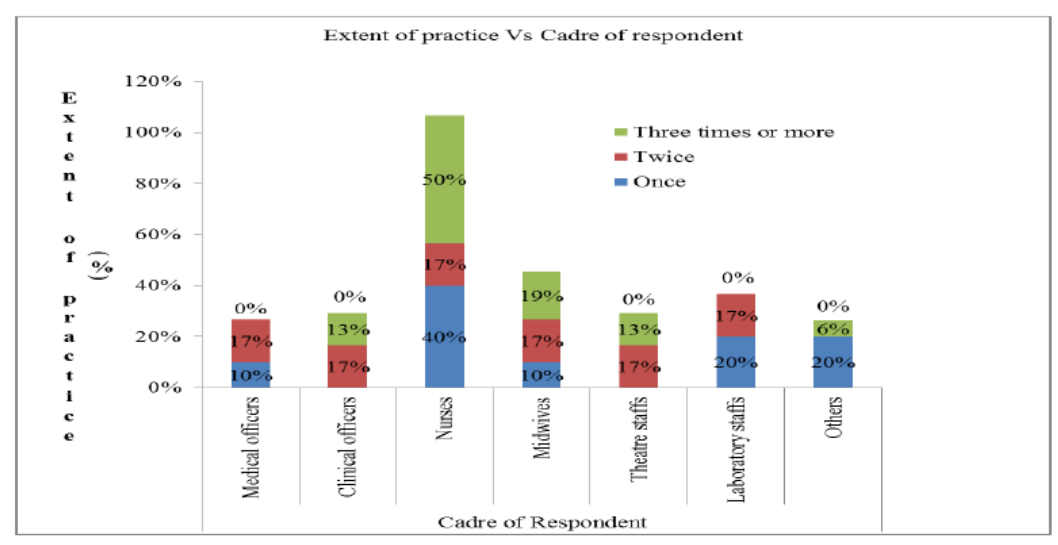

Figure 7: Cross tabulation of extent of practice over 5year period versus cadre of respondent

Figure 7 above shows that the greatest categories of staffs who practiced medical incident reporting three times or more in a 5year period were nurses and midwives, constituting $50 \%$ and $19 \%$ respectively. Therefore, being a nurse or a midwife in Midigo health centre IV gives a healthcare professional better chance of practicing medical incident reporting. However, the researcher is 95\% confident that this finding was by chance, $\mathrm{X} 2(12, \mathrm{~N}=44)=11.64, p=.48$. In other word, there was no significant relationship between the extent to which one practiced medical incident reporting and cadre of the said respondent.

Relationship between knowledge about medical incident reporting and extent of practice over a 5year period: The researcher, again, went ahead to examine the relationship between knowledge about medical incident reporting and extent of practice of medical incident reporting over a 5year period. Cross tabulation of knowledge about reporting and extent of practice was done and the result was as shown in figure 8 below 


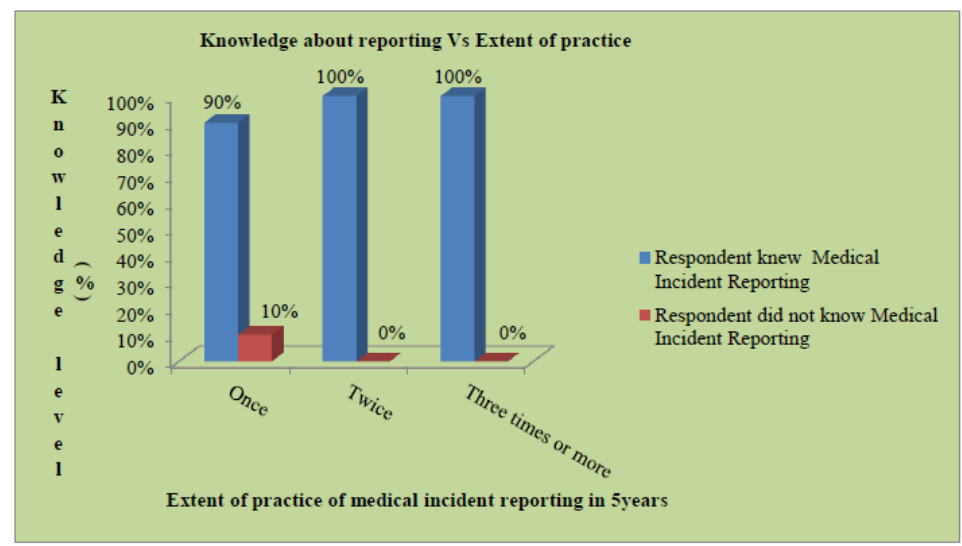

Figure 8: Knowledge of medical incident reporting versus extend of practice

Figure 8 shows that under the category of those who practiced (participated in) medical incident reporting once in 5years, only $10 \%$ did not know medical incident reporting. $90 \%$ of this category of respondents knew about the subject matter. For the categories that practiced (participated in) more than once, they all knew medical incident reporting. Much as knowledge on medical incident reporting appears to be high with increase in the extent of practice, there was no evidence to justify this trend, $X 2(2, N=44)=2.27, p=.32$.

\section{Factors affecting medical incident reporting in Midigo Health Centre IV}

The factors affecting medical incident reporting were listed in two categories. The first categories were those factors which facilitated the respondent to participate in reporting over the last 5years. The second categories were those factors which hindered the respondent from participation over a 5year period. These were called barriers, by the researcher. The respondent was allowed to select any many factors as possible for the respective category he/she falls.

Factors that facilitated the practice of affecting medical incident reporting: The factors which facilitated the practice varied from one respondent to the next. They were summarized in table 4 below;

Table 4: Factors that facilitated the practice of medical incident reporting

\begin{tabular}{lll}
\hline Factors for participation & Frequency & Percentage \\
\hline $\begin{array}{l}\text { Respondent was Knowledgeable(educated) about } \\
\text { reporting }\end{array}$ & 23 & $71.9 \%$ \\
$\begin{array}{l}\text { Respondent had strong positive feeling to participate and } \\
\text { improve patient safety }\end{array}$ & 26 & $81.3 \%$ \\
$\begin{array}{l}\text { Practice(Culture) of reporting was high in the facility } \\
\begin{array}{l}\text { Respondent was able to get feedback about reported } \\
\text { incidents }\end{array}\end{array}$ & 6 & 11 \\
\hline
\end{tabular}

Total percentage $>100 \%$ because of multiple responses

From table 4 above, out of the $72.7 \%$ of the respondents (reported earlier) who practice incident reporting over a 5year period, the majority (81.3\%) practiced because they had strong feeling to improve patient safety. Therefore, most of the staffs of Midigo health centre IV participated in incident reporting either because strong feeling to improve patient safety or because they were knowledgeable (educated) about reporting (71.9\%). The institutional high culture of reporting and ability to get feedback of reported incidents did not have much impact in their participation.

Barriers to the practice of medical incident reporting over a 5year period: Again, the factors which acted as barriers to the practice of medical incident reporting varied from one respondent to the next. They were summarized in table 5 below; 
Table 5: Barriers to the practice of medical incident reporting

\begin{tabular}{lll}
\hline Barriers & Frequency & Percentage \\
\hline Respondent didn't know how and where to report & 8 & $66.7 \%$ \\
The practice(culture) of reporting was weak(low) & 5 & $41.7 \%$ \\
Fear of consequence of reporting & 0 & $0 \%$ \\
Respondent lacked time to report & 3 & $25 \%$ \\
Other factors & 2 & $16.7 \%$ \\
\hline
\end{tabular}

Total percentage $>100 \%$ because of multiple responses

Table 5 above shows that out of the $27.3 \%$ of respondent who did not participate in medical incident reporting, the majority (66.7\%) did not do so because they didn "t know how and where to report. The practice (culture) of weak institutional reporting also featured as a great barrier, standing at $41.7 \%$. The fear of the consequence of reporting did not contribute in any way to their non-participation in the 5year period.

\section{Discussions, conclusion and recommendations}

\section{Introduction}

This chapter displays the discussion on the findings, conclusion based on this study and recommendation to readers or stakeholders to this research work.

\section{Discussion}

The discussions have been sub-divided into four result areas in accordance with the specific objectives of this study.

Knowledge about medical incident reporting among healthcare professionals: Being knowledgeable about something often influences one"s judgment whether or not be active. Similarly, it is expected that where healthcare professionals are more knowledgeable about medical incident reporting, the practice must be high. In fact Jansma et al. (2011) argued that patient safety education can have immediate and long-term positive effects on knowledge, skills and attitudes, and modestly influence the reporting behavior of resident healthcare professionals.

The finding in this study also showed that the extent of practice of medical incident reporting was highest among those healthcare professionals whose knowledge level was $100 \%$. This is consistent with other studies, including World Health Organization (WHO). WHO argued that for several reasons, the effectiveness of incident reporting is limited. One of such reasons was related to knowledge gap, that is, lack of understanding of what a reportable incident is (WHO, 2009).

Attitude of healthcare professionals towards medical incident reporting: The attitude, in many cases goes hand-in-hand with knowledge deficit on the subject matter, although there may be other aggravating factors. For example, many researchers have found that fear of being punished in case of reported medical incident has negatively affected the practice of reporting in many healthcare settings (Radhakrishna, 2016 and WHO, 2009).

However, in Midigo health centre IV, this factor did not contribute at all to any negative practice. No respondent healthcare professional was able to say he/she did not participate in reporting because he/she feared being punished. The knowledge level of healthcare professionals in Midigo was reasonably high

(84.1\%) and this was coupled with very strong positive attitude (97.7\%) towards medical incident reporting, hence level of practice being $72.7 \%$. Therefore, in terms of fear of being punished for medical incidents, this study was not consistent with other studies.

The practice and the extent to which medical incident reporting is practiced: The extent of incident reporting depended on the level of knowledge and positive attitudes towards patient safety concept, among healthcare professionals. This is consistent with other studies. For that matter, therefore, the more someone is educated on patient safety, the more 
Texila International Journal of Medicine

Volume 4, Issue 2, Dec 2016

the ability and willingness to report incidents. In fact, positive changes in knowledge, skills and attitudes would be paramount after such education course (Jansma et al., 2011)

Factors affecting medical incident reporting: According to Marilyn et al. (2004), the common barriers to reporting incidents included; time constraints, unsatisfactory processes, deficiencies in knowledge, cultural norms, inadequate feedback, beliefs about risk and a perceived lack of value in the process.

In the context of Midigo Health Centre IV, the commonest barriers were; respondents didn't know how and where to report, weak institutional culture of reporting and to a lesser extent the perceived lack of time to report. These factors are not any different from those found by other researchers. Uniquely speaking, fear of punishment following medical incident reporting did not act as a barrier at all in Midigo.

\section{Conclusion}

Medical incident reporting still remains a key tool in improving patient safety. The greater the practice of reporting, the better; as evident by the strong positive feeling towards medical incident reporting, in this study. Educating professionals on incident reporting and strong positive individual feeling to improve safety have remained the major factors facilitating medical incident reporting. Likewise, lack of knowledge on where and how to report, coupled with weak institutional culture of reporting have remained the major barriers to practicing medical incident reporting.

\section{Recommendations}

Following the findings from this study, the researcher would like to make the following recommendations;

I. Let many more researches be done on medical incident reporting in other health facilities, in Yumbe district and beyond, to assess their status regarding such reporting.

II. Let continuous professional development (CPD) / continuous medical education (CME) be done in Midigo health centre IV and other healthcare facilities in and around Yumbe district. This will help to increase patient safety awareness among healthcare professionals.

III. Let healthcare professionals be encouraged and fully facilitated by management of healthcare facilities, district health office (DHO) and development partners such as Calvary Chapel of Midigo, to conduct of healthcare researches in their institutions. This will help to identify and correct problem areas.

\section{Acknowledgement}

I do sincerely acknowledge the contributions of everybody towards the successful completion of this research work. In particular, I want to recognize the supervisory role played by Dr Yayi Alfred, the District Health Officer (DHO) of Yumbe district local government who has been my professional supervisor throughout the course of this research work. I also acknowledge the supervisory roles played by Pastor Jjagwe Charles, the Chief Executive Officer (CEO) of Calvary Chapel of Midigo who doubles as a Public Health Specialist and my immediate supervisor. May God bless them all!

\section{References}

\section{Book}

[1.] Ministry of Health Uganda. (2015). Annual Health Sector Performance Report: Financial Year 2014/2015. Kampala: Ministry of Health Uganda.

\section{Journal article}

[2.] Mahajan, R. P. (2010). Critical incident reporting and learning. British Journal of Anaesthesia,105 (1), 69-75. doi:10.1093/bja/aeq133 


\section{Project report}

[3] Nabudere, H., Asiimwe, D. and Semakula, D. (2014). Improving patient safety for better quality of care. Regional East African Community Health (REACH) Policy Initiative

\section{Journal article}

[4] Anderson, J. E., Naonori, K., Rhiannon, W., and Anneliese, D. (2013). Can incident reporting improve safety? Healthcare practitioners" views of the effectiveness of incident reporting. International Journal for Quality in Health Care, 2013(25), 141-150. doi: 10.1093/intqhc/mzs081

\section{Journal article}

[5] Lawton, R. and Parker, D. (2002). Barriers to incident reporting in a healthcare system. Qual Saf Health Care,11, 15-18. doi: 10.1136/qhc.11.1.15

\section{Journal article}

[6] Evans, S. M., Berry, J. G., Smith, B. J., Esterman, A., Selim, P., O "Shaughnessy, J. and DeWit, M. (2006). Attitudes and barriers to incident reporting: a collaborative hospital study. Qual Saf Health Care, 15, 39-43. doi: 10.1136/qshc.2004.012559

\section{Journal article}

[7] Omona, K. (2015). Patients and Health Workers' Engagement in Patient Safety in Healthcare in Kitgum General Hospital. South American Journal of Management, 1, 1-44.

\section{Journal article}

[8] Domenico, F., Paolo, R., Aida, B., Claudia, P., and Maria, P., (2012). Patient Safety and Medical Errors: Knowledge, Attitudes and Behavior among Italian Hospital Physicians. International journal for quality in healthcare, 2012, 1-8.

\section{Journal article}

[9] Jansma, J. D., Cordula, W., Reinier, W. t. K., \& Arnold, B. B. (2011). Effects on incident reporting after educating residents in patient safety: a controlled study. BMC Health Services Research, 11:335. doi:10.1186/1472-6963-11-335

\section{Article from a reference book}

[10] World Health Organization. (2009). Glossary of Patient Safety Concepts and References. In WHO, World Alliance for Patient Safety Taxonomy (Technical Annex II). Geneva: World Health Organization.

London, UK: Wiley Blackwell.

\section{Journal article}

[11] Kreckler, S., Catchpole, K., McCulloch, P. and Handa, A. (2009). Factors influencing incident reporting in surgical care. Qual Saf Health Care, 18, 116-120 doi:10.1136/qshc.2008.026534

\section{Journal article}

[12] Radhakrishna, S. (2016). Culture of blame in the National Health Service; consequences and solutions. British Journal of Anesthesia, 115, 653-655.

\section{Journal article}

[13] Anja, H., Brunsveld-Reinders, M., Sesmu, A., Rien, D. V. and Evert, D. J. (2015). Incident and error reporting systems in intensive care: a systematic review of the literature. Oxford University Press, 2015, 2-13. doi: http://dx.doi.org/10.1093/intqhc/mzv100

\section{Web page}

[14] Patient Safety Network. (2014). Voluntary Patient Safety Event Reporting (Incident Reporting). Retrieved from https://psnet.ahrq.gov/primers/primer/13/voluntary-patient-safety-event-reportingincident-reporting 
Texila International Journal of Medicine

Volume 4, Issue 2, Dec 2016

\section{Book}

[15] Vincent, C. (2011). The Essentials of Patient Safety, London, UK: Wiley Blackwell.

\section{Journal article}

[16] Gifford, M., L. and Anderson, J., E. (2010). Barriers and Motivating Factors in Reporting Incidents of Assault in Mental Health Care. Journal of the American Psychiatric Nurses Association, 16(5), 288-298. doi: 10.1177/1078390310384862

\section{Journal article}

[17] Naonori, K. and Anneliese, D. (2008). Factors Affecting Willingness to Report Patient Safety Incidents in Hospitals. National Institute for Health Research, 1, 1-38.

\section{Journal article}

[18] Polisena, J., Gagliardi, A., Urbach, D., Tammy, C. and Michelle, F. (2015). Factors that influence the recognition, reporting and resolution of incidents related to medical devices and other healthcare technologies: a systematic review. BioMed Central (2015), 1-11. doi:10.1186/s13643-015-0028-0

\section{Journal article}

[19] Haller, G., Courvoisier, D. S., Anderson, H. and Myles, P. S. (2011). Clinical factors associated with the non-utilization of an anaesthesia incident reporting system. British Journal of Anaesthesia, 107 (2), 171-179. doi:10.1093/bja/aer148

\section{Journal article}

[20] Julius, C. P., Thierry, G. and Peter, J. P. (2013). What to do with healthcare Incident Reporting Systems. Journal of Public Health Research 2013, 2(27), 154-159. doi:10.4081/jphr.2013.e27

\section{Journal article}

[21] Marilyn, J. K., Sue, M. E., Brian, J. S. and Jesia, G. B. (2004). Attitudes of doctors and nurses towards incident reporting: a qualitative analysis. MJA, 36-39.

\section{Journal article}

[22] Hooper, P., Kocman, D., Carrl, S. and Tarrant, C. (2015). Junior doctors" views on reporting concerns about patient safety: a qualitative study. British Medical Journal, 91, 251-256. doi:10.1136/postgradmedj-2014-133045

\section{Book}

[23] Uganda Bureau of Statistic. (2015). National Population and Housing Census 2014. Kampala: Uganda Bureau of Statistic (UBOS). 